\title{
Intestinal Farnesoid X Receptor and Takeda G Protein Couple Receptor 5 Signaling in Metabolic Regulation
}

\author{
John Y.L. Chiang Preeti Pathak Hailiang Liu Ajay Donepudi Jessica Ferrell \\ Shannon Boehme \\ Department of Integrative Medical Sciences, Northeast Ohio Medical University, Rootstown, Ohio, USA
}

\section{Keywords}

Bile acid synthesis · Cholesterol 7a-hydroxylase · Diabetes ·

Drug therapy $\cdot$ Non-alcoholic fatty liver disease

\begin{abstract}
Bile acids play a critical role in the regulation of glucose, lipid and energy metabolisms by activating the nuclear bile acid receptor farnesoid $X$ receptor (FXR) and membrane $G$ protein-coupled bile acid receptor-1 (aka takeda $G$ protein couple receptor 5, TGR5) signaling. Paradoxical roles of FXR in the regulation of glucose and lipid metabolism and metabolic disorder have been reported recently. The activation or inhibition of intestinal FXR signaling has been shown to improve insulin and glucose sensitivity and energy metabolism to prevent diabetes, obesity and non-alcoholic fatty liver disease (NAFLD). TGR5 has an anti-inflammatory function in the intestine and stimulates glucagon-like peptide-1 (GLP-1) secretion in the intestine to stimulate insulin secretion from the pancreas. The role of TGR5 in metabolism and metabolic regulation is not clear and warrants further study. FXR and TGR5 are co-expressed in the ileum and colon. These 2 bile acid-activated receptors may cooperate to stimulate GLP-1 secretion and improve hepatic metabolism. FXR and TGR5 dual agonists may have therapeutic potential for treating diabetes and NAFLD.

(c) 2017 S. Karger AG, Basel
\end{abstract}

() 2017 S. Karger AG, Basel

E-Mail karger@karger.com

www.karger.com/ddi

\section{Background}

Bile Acid Synthesis

It is now well established that bile acids are signaling molecules that regulate lipid, glucose and energy homeostasis through the activation of the bile acid nuclear receptor, farnesoid X receptor (FXR) and the membrane G-protein-coupled bile acid receptor 1 (Gpbar-1, aka takeda G protein couple receptor 5, TGR5) [1]. Bile acid synthesis in the liver generates 2 primary bile acids, cholic acid (CA) and chenodeoxycholic acid (CDCA; fig. 1) [2]. In mouse liver, CDCA is converted to $\alpha$ - and $\beta$-muricholic. Cholesterol 7a-hydroxylase (CYP7A1) is the first rate-limiting enzyme in the classic bile acid synthesis pathway. Sterol $12 \alpha$-hydroxylase (CYP8B1) catalyzes CA synthesis. Mitochondrial sterol 12a-hydroxylase (CYP27A1) catalyzes steroid side-chain oxidation, followed by side-chain cleavage reaction in the peroxisomes to cleave $2 \mathrm{C}$-units from the side-chain to form C24 bile acids. In the alternative pathway, CYP27A1 initiates bile acid synthesis, followed by oxysterol $7 a$-hydroxylase (CYP7B1) in most tissues. The oxysterol intermediates formed are transported to the liver for synthesis of mainly CDCA. Bile acids are re-conjugated to glycine and taurine and secrete into bile, store in the gallbladder and secreted into the intestinal tract after a meal to facilitate intestinal absorption of fats and lip- 


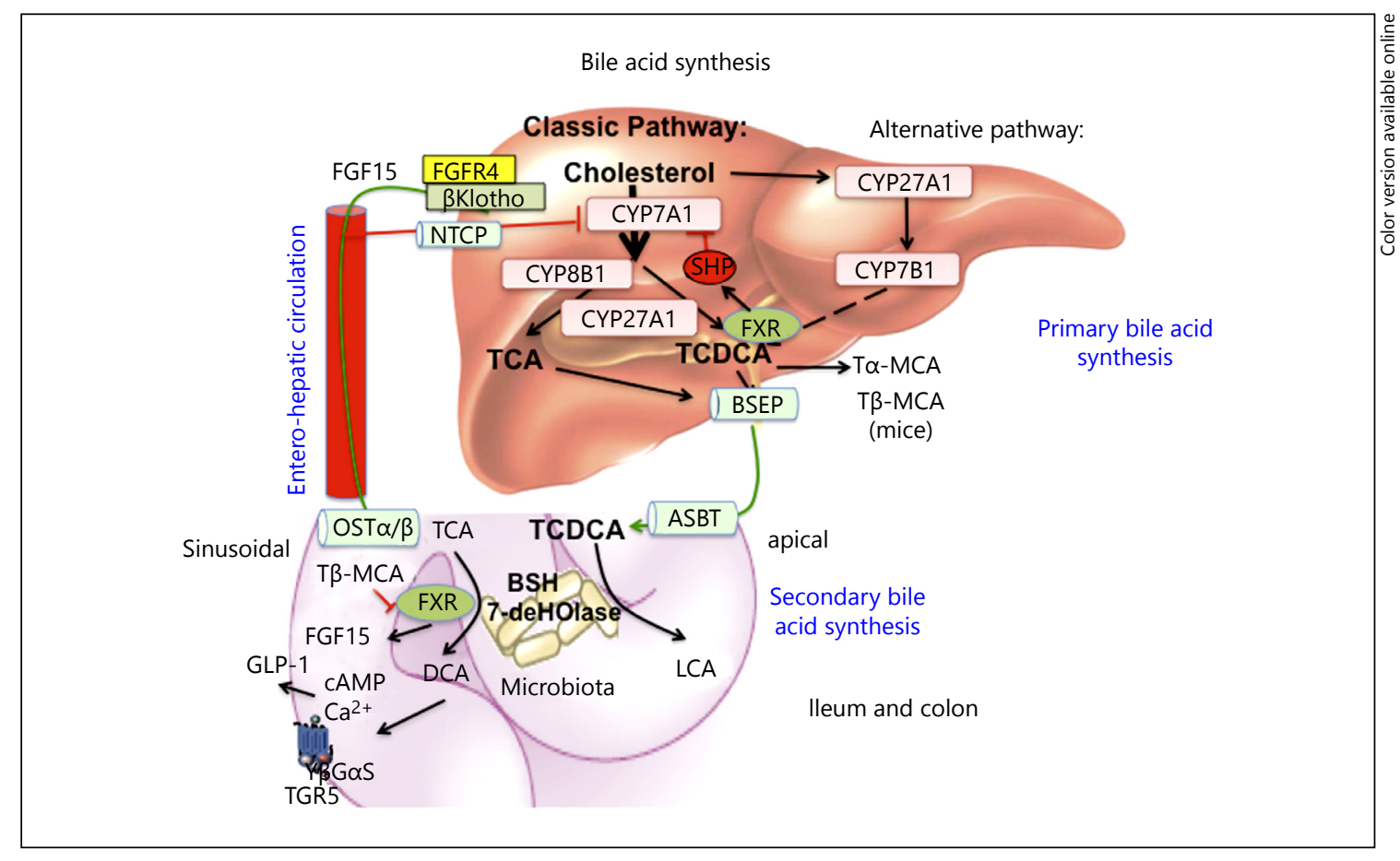

Fig. 1. Bile acid synthesis and regulation. Two bile acid synthesis pathways exist in the liver. The classic pathway is initiated by CYP7A1, while the alternative pathway is initiated by sterol 27-hydroxylase (CYP27A1). In humans, 2 primary bile acids are synthesized: CA and CDCA. For synthesis of CA, CYP8B1 is needed. Mitochondrial CYP27A1 catalyzes the steroid side-chain oxidation reaction. In the alternative pathway, CYP7B1 is involved in the synthesis of oxysterol intermediates, which are converted to bile acids, mainly CDCA, in hepatocytes. In mice, CDCA is converted to tauro- $\alpha$-muricholic acid and then T $\beta$ MCA as primary bile acids. Bile acids are secreted through the bile salt export pump, stored in the gallbladder, and secreted into the gastrointestinal tract and reabsorbed through apical sodium-dependent bile salt transporter in the ileum. In the colon, TCA and TDCA are first de-conjugated by bacterial $\mathrm{BSH}$, and dehydroxylated by bacterial 7a-dehydroxylases to form the secondary bile acids, DCA and LCA, respectively. In the ileum, the bile acid efflux transporter, sinusoidal organic solute transporter $\alpha / \beta$ effluxes bile acids and

id-soluble vitamins. In the intestine, gut microbial bile salt hydrolases (BSH) deconjugate taurine- and glycine-conjugated-bile acids, and bacterial $7 \alpha$-dehydroxylases convert primary bile acids CA and CDCA to deoxycholic acid (DCA) and lithocholic acid (LCA), respectively, which are re-conjugated to glycine or taurine.

\section{Regulation of Bile Acid Synthesis}

Bile acids are the end products of cholesterol catabolism in the liver. Bile acids facilitate biliary cholesterol and lipid secretion to form mixed micelles in the gallbladder. circulates bile acids to the liver via the portal vein to inhibit bile acid synthesis. Two mechanisms have been suggested to inhibit CYP7A1 and CYP8B1 gene transcription. In the liver, bile acids activate nuclear receptor FXR to induce a negative nuclear receptor, SHP to inhibit CYP7A1 and CYP8B1 gene transcription. In the intestine, FXR induces FGF15, or FGF19 in humans, which is circulated to the liver to activate plasma membrane FGF receptor 4 (FHFR4)/3Klotho signaling to inhibit CYP7A1 gene transcription. TGR5 (Gpbar-1) is expressed in the ileum and colon. TGR5 activates cAMP signaling to stimulate GLP-1 secretion in enteroendocrine L cells. GLP-1 stimulates insulin secretion in pancreatic $\beta$ cells to improve insulin sensitivity. Decreasing BSH activity in gut microbiota increases T $\beta M C A$, which antagonizes FXR activity to reduce FGF15/19 and stimulates bile acid synthesis in hepatocytes. Increasing BSH activity in gut microbiota reduces TMCA antagonism of FXR and stimulates FGF15/19 production to inhibit bile acid synthesis. Thus, the gut-to-liver axis plays a critical role in the regulation of bile acid synthesis and metabolic homeostasis.

Bile acids are reabsorbed in the ileum and transported via enterohepatic circulation (EHC) to the liver to inhibit bile acid synthesis by transcriptional repression of CYP7A 1 and $C Y P 8 B 1$ gene expression. EHC is highly efficient, occurs several times a day and recycles about $95 \%$ bile acids. EHC of bile acids facilitate absorption of nutrients to the liver to be metabolized and redistributed to other tissues. Two mechanisms have been suggested for bile acid feedback regulation of bile acid synthesis. In the liver, bile acids activate FXR to induce a negative nuclear receptor small heterodimer partner (SHP) to inhibit CYP7A1 and 
CYP8B1 gene transcription. In the intestine, FXR induces fibroblast growth factor 15 (FGF15), which is transported to the liver to activate hepatic FGF receptor 4 signaling to inhibit CYP7A1 gene transcription.

The gut microbiota plays a critical role in pathogen defense, immunity and nutrient harvest. Bile acids inhibit gut bacterial overgrowth and the gut microbiota controls bile acid metabolism by the de-conjugation of bile acids by BSH and de-hydroxylation by $7 \alpha$-dehydroxylases, thus controlling bile acid composition in the pool $[3,4]$. The gut microbiota utilizes short chain fatty acids from dietary carbohydrates for energy metabolism and regulates glucose, lipid and energy metabolism in the liver, muscle and adipocytes. The gut microbiota also controls gut hormones/peptides release from the intestine, such as YY and glucagon-like peptide-1 (GLP-1). These hormones regulate insulin secretion from pancreatic $\beta$ cells and in turn, glucose homeostasis. Dysbiosis has been associated with inflammatory bowel diseases, obesity and type 2 diabetes and non-alcoholic fatty liver disease (NAFLD), cirrhosis and liver cancer [5-8].

Bile acid synthesis is regulated by circadian rhythms and the fasting and refeeding cycle [1,9]. In mice, CYP7A 1 and bile acid synthesis peaks right after entering the dark cycle and decreases in the midnight. In humans, bile acid synthesis peaks at 9 a.m. and midafternoon [1012]. Circadian rhythms are influenced by feeding and diets [13-15]. CYP7A1 expression rapidly increases after feeding and decreases after fasting, while CYP8B1 is stimulated by fasting and decreased by feeding [16]. Thus, circadian expression pattern of these 2 regulatory enzymes determines the rate of bile acid synthesis and bile acid composition in the pool. Disruption of the circadian regulation of bile acid synthesis alters hepatic metabolism and homeostasis and contributes to diabetes and obesity [17].

\section{Bile Acid Receptor Regulation of Hepatic Metabolism}

Bile acids regulate hepatic metabolism through the activation of FXR and TGR5 signaling. The role of FXR in the regulation of hepatic lipid and glucose metabolism has been studied extensively. The activation of FXR by bile acids and agonists reduces serum triglycerides and improves glucose tolerance and insulin resistance in diabetic mice $[18,19]$. However, more recent studies show the opposite effect in that deficiency of FXR improves glucose homeostasis [20] and the activation of FXR lowers bile acid synthesis and induces obesity and diabetes [21]. Moreover, several recent studies show that deficiency of intestinal Fxr gene or antagonism of intestine FXR by the antioxidant tempol or intestine-selective FXR inhibitor Gly-MCA increases TMCA to antagonize intestinal FXR and decrease obesity and NAFLD [22-24]. Conversely, the activation of intestinal FXR by a selective FXR agonist fexaramine (FEX) also improves diabetes and obesity in mice [25]. Thus, the role and mechanism of FXR signaling in the regulation of lipid and glucose metabolism is controversial and not completely understood.

The role of TGR5 in the regulation of hepatic metabolism has not been studied in depth. TGR5 is widely expressed in many tissues including intestine, gallbladder, liver, and brain [26-28]. In the liver, TGR5 is expressed in Kupffer cells and sinusoidal endothelial cells, but not in hepatocytes $[28,29]$. In the gastrointestinal tract, the activation of TGR 5 by bile acids and agonists protects intestinal barrier function, reduces inflammation, and stimulates gallbladder refilling and GLP-1 secretion from enteroendocrine L cells [30], which increases postprandial insulin secretion from pancreatic $\beta$ cells and improves insulin resistance [31]. GLP-1 is an intestinal incretin produced in L cells through the processing of preproglucagon by prohormone convertase $1 / 3(\mathrm{PC} 1 / 3)$ and is released in response to meal intake [32]. GLP-1 secretion is stimulated by nutrients in the intestinal lumen, such as carbohydrates, fats and proteins. The synthetic GLP-1 analog exendin-4 reduces hepatic steatosis by decreasing lipogenesis and inducing fatty acid oxidation [33]. The activation of TGR5 induces thyroid hormone deiodinase 2 to stimulate energy metabolism in brown adipose tissues [34]. The activation of TGR5 stimulates adenylyl cyclase to convert AYP to cAMP, which activates protein kinase A to activate the cAMP response elementbinding protein (CREB) and results in alleviation of obesity and hepatic steatosis in diet-induced obese mice [31]. Thus, further study of the role of FXR and TGR5 in hepatic metabolism and diabetes is needed.

\section{Key Messages}

FXR and TGR5 are co-expressed in intestinal endocrine L cells. It is possible that the activation of FXR may stimulate GLP-1 secretion from $\mathrm{L}$ cells and contribute to increasing insulin secretion from the pancreatic $\beta$ cell to improve insulin sensitivity. To test this hypothesis, FXR and TGR5 agonists were used to study their effects on GLP-1 secretion in wild type, $\mathrm{Fxr}^{-/-}$and $\mathrm{TgrF}^{-/-}$mice, and in enteroendocrine GLUTag and STC- 1 cells. Oral gavage of FXR agonists OCA, GW4064 and FEX, and TGR5 selective agonist INT777 agonist stimulated GLP-1 secretion in wild type mice and GLUTag cells. Real-time PCR assay showed that the 4 TGR5 mRNA expression level was induced in the intestine of wild type mice, but reduced in $\mathrm{Fxr}^{-/-}$mice, suggesting that FXR induced 
TGR5 gene transcription. Interestingly, mRNA levels of PC1/3, which splices preproglucagon to glucagon, were also induced by OCA and GW4064 and FEX, and reduced in $\mathrm{Fxr}^{-/-}$mice. Immunoblot analysis showed OCA and FEX induced TGR5 and PC1/3 protein levels in mouse ileum. CREB reporter assay and site-directed mutagenesis identified an FXR response element located in the proximal promoter of human and mouse Tgr 5 gene promoter. Electrophoretic mobility shift assay demonstrated FXR/RXR binding to this putative FXRE. Chromatin immunoprecipitation assay demonstrated the occupancy of FXR/RXR and co-activator PGC-1 $1 \alpha$ to the TGR5 promoter region containing the FXRE.

To further confirm the role of FXR in GLP-1 secretion, a GLP-1 secretion assay was performed in $\mathrm{Fxr}^{-/-}$, and $\mathrm{Tgr5}^{-/-}$mice. In $\mathrm{Tgr}^{-/-}$mice, GLP-1 secretion was reduced $\sim 50 \%$, indicating that some other factor (FXR) was also involved in GLP-1 secretion. Oral gavage of FEX did not affect GLP-1 secretion in $\mathrm{Tgr5}^{-/-}$mice indicating that TGR5 was required for GLP-1 secretion. In $\mathrm{Fxr}^{-/-}$ mice, GLP-1 secretion was reduced $\sim 50 \%$ because TGR5 expression was reduced in the absence of FXR. This study reports for the first time that the activation of FXR induces $\operatorname{Tgr} 5$ and $P c 1 / 3$ gene transcription to stimulate GLP-1 processing and secretion from intestinal L cells during the postprandial state. FXR and TGR5 may coordinately regulate GLP-1 production and secretion.

Our data also showed that OCA and FEX have differential effects on hepatic glucose and lipid metabolism; FEX is more effective in inhibiting gluconeogenesis and improving glucose tolerance, while OCA is more effective in stimulating fatty acid oxidation and improving lipid metabolism. This study also indicated that GLP-1 may directly stimulate insulin-like signaling in hepatocytes to inhibit gluconeogenesis and improve insulin and glucose sensitivity. FEX inhibits hepatic gluconeogenic gene expression and improves insulin and glucose tolerance and reduced serum cholesterol and hepatic triglycerides in diabetic $\mathrm{db} / \mathrm{db}$ mice.

\section{Conclusions}

The gut-to-liver axis plays a critical role in regulating bile acid synthesis and FXR and TGR5 signaling in liver and intestine. The activation of FXR by bile acids in the intestine induces FGF15 to inhibit bile acid synthesis in the liver, whereas the inhibition of BSH by gut bacteria or the antioxidant tempol increases T- $\beta$-MCA, which may antagonize FXR and FGF15 production to stimulate bile acid synthesis $[22,35]$. In mice deficient of intestinal Fxr, FGF15 production is reduced and bile acid synthesis is upregulated to stimulate hepatic FXR and improve glucose and insulin sensitivity, and thus prevent diet-induced NAFLD [23]. The intestine-selective inhibition of FXR by Gly-MCA also reduces intestine ceramide production and contributes to improving diabetes and obesity [24]. Disruption of the gut-to-liver axis by deleting or inhibiting intestinal FXR or treating with antibiotics, alters gut microbiota to increase ileum TMCA and bile acid synthesis to regulate glucose and lipid metabolism and prevent diet-induced diabetes and obesity. On the other hand, pharmacological activation of intestinal FXR may reduce TCA and increase LCA to stimulate FGF15 and GLP-1 production to improve hepatic metabolism and insulin and glucose sensitivity. Intestinal FXR and TGR5 signaling crosstalk may coordinately stimulate GLP-1 secretion to improve hepatic glucose and insulin sensitivity in diabetes. FXR and TGR5 dual agonists may be effective for treating liver-related metabolic diseases, diabetes and NAFLD.

\section{Disclosure Statement}

The author has nothing to disclose.

\section{Financial Support}

This study is supported by NIH grants DK44442 and DK58379.

\section{References}

1 Li T, Chiang JY: Bile acid signaling in metabolic disease and drug therapy. Pharmacol Rev 2014;66:948-983.

2 Chiang JY: Bile acids: regulation of synthesis. J Lipid Res 2009;50:1955-1966.

3 Inagaki T, et al: Regulation of antibacterial defense in the small intestine by the nuclear bile acid receptor. Proc Natl Acad Sci U S A 2006; 103:3920-3925.

4 Jones BV, et al: Functional and comparative metagenomic analysis of bile salt hydrolase activity in the human gut microbiome. Proc Natl Acad Sci U S A 2008;105:13580-13585.

5 Aron-Wisnewsky J, et al: Gut microbiota and non-alcoholic fatty liver disease: new in- sights. Clin Microbiol Infect 2013;19:338348.

6 Kakiyama G, et al: Modulation of the fecal bile acid profile by gut microbiota in cirrhosis. J Hepatol 2013;58:949-955.

7 Joyce SA, Gahan CG: The gut microbiota and the metabolic health of the host. Curr Opin Gastroenterol 2014;30:120-7.

8 Ridlon JM, et al: Bile acids and the gut microbiome. Curr Opin Gastroenterol 2014;30: 332-338.

9 Chiang JY, Miller WF, Lin GM: Regulation of cholesterol 7 alpha-hydroxylase in the liver. Purification of cholesterol 7 alpha-hydroxylase and the immunochemical evidence for the induction of cholesterol 7 alpha-hydroxylase by cholestyramine and circadian rhythm. J Biol Chem 1990;265:3889-3897.

10 Ferrell JM, Chiang JY: Circadian rhythms in liver metabolism and disease. Acta Pharm Sin B 2015;5:113-122.

11 Galman C, Angelin B, Rudling M: Bile acid synthesis in humans has a rapid diurnal variation that is asynchronous with cholesterol synthesis. Gastroenterology 2005;129:1445-1453.

12 Lundasen $T$, et al: Circulating intestinal fibroblast growth factor 19 has a pronounced diurnal variation and modulates hepatic bile acid synthesis in man. J Intern Med 2006;260:530536 
13 Asher G, Schibler U: Crosstalk between components of circadian and metabolic cycles in mammals. Cell Metab 2011;13:125-137.

14 Vollmers C, et al: Time of feeding and the intrinsic circadian clock drive rhythms in hepatic gene expression. Proc Natl Acad Sci U S A 2009;106:21453-21458.

15 Hatori M, et al: Time-restricted feeding without reducing caloric intake prevents metabolic diseases in mice fed a high-fat diet. Cell Metab 2012;15:848-860.

16 Pathak P, Li T, Chiang JY: Retinoic acid-related orphan receptor a regulates diurnal rhythm and fasting induction of sterol $12 \alpha$-hydroxylase in bile acid synthesis. J Biol Chem 2013;288:37154-37165.

17 Ferrell JM, Chiang JY: Short-term circadian disruption impairs bile acid and lipid homeostasis in mice. Cell Mol Gastroenterol Hepatol 2015;1:664-677.

18 Watanabe $\mathrm{M}$, et al: Bile acids lower triglyceride levels via a pathway involving FXR, SHP, and SREBP-1c. J Clin Invest 2004;113:14081418.

19 Zhang Y, et al: Activation of the nuclear receptor FXR improves hyperglycemia and hyperlipidemia in diabetic mice. Proc Natl Acad Sci U S A 2006;103:1006-1011.

20 Prawitt J, et al: Farnesoid X receptor deficiency improves glucose homeostasis in mouse models of obesity. Diabetes 2011;60:18611871.
21 Watanabe $\mathrm{M}$, et al: Lowering bile acid pool size with a synthetic farnesoid $\mathrm{X}$ receptor (FXR) agonist induces obesity and diabetes through reduced energy expenditure. J Biol Chem 2011;286:26913-26920.

22 Li F, et al: Microbiome remodelling leads to inhibition of intestinal farnesoid X receptor signalling and decreased obesity. Nat Commun 2013;4:2384.

23 Jiang C, et al: Intestinal farnesoid X receptor signaling promotes nonalcoholic fatty liver disease. J Clin Invest 2015;125:386-402.

24 Jiang $C$, et al: Intestine-selective farnesoid X receptor inhibition improves obesity-related metabolic dysfunction. Nat Commun 2015;6: 10166.

25 Fang S, et al: Intestinal FXR agonism promotes adipose tissue browning and reduces obesity and insulin resistance. Nat Med 2015 21:159-165.

26 Maruyama $\mathrm{T}$, et al: Identification of membrane-type receptor for bile acids (M-BAR). Biochem Biophys Res Commun 2002;298: 714-719.

27 Kawamata Y, et al: A G protein-coupled receptor responsive to bile acids. J Biol Chem 2003;278:9435-9440.
28 Keitel V, et al: Expression and function of the bile acid receptor TGR5 in Kupffer cells. Biochem Biophys Res Commun 2008;372:78-84.

29 Keitel V, et al: The G-protein coupled bile salt receptor TGR5 is expressed in liver sinusoidal endothelial cells. Hepatology 2007;45:695704

30 Katsuma S, Hirasawa A, Tsujimoto G: Bile acids promote glucagon-like peptide-1 secretion through TGR5 in a murine enteroendocrine cell line STC-1. Biochem Biophys Res Commun 2005;329:386-390.

31 Thomas C, et al: TGR5-mediated bile acid sensing controls glucose homeostasis. Cell Metab 2009;10:167-177.

32 Mojsov S, et al: Preproglucagon gene expression in pancreas and intestine diversifies at the level of post-translational processing. J Biol Chem 1986;261:11880-11889.

33 Ding $X$, et al: Exendin-4, a glucagon-like protein-1 (GLP-1) receptor agonist, reverses hepatic steatosis in ob/ob mice. Hepatology 2006;43:173-181.

34 Watanabe $M$, et al: Bile acids induce energy expenditure by promoting intracellular thyroid hormone activation. Nature 2006;439: 484-489.

35 Sayin SI, et al: Gut microbiota regulates bile acid metabolism by reducing the levels of tauro-beta-muricholic acid, a naturally occurring FXR antagonist. Cell Metab 2013;17: 225-235. 